\title{
Perpetual Robot Swarm: Long-Term Autonomy of Mobile Robots Using On-the-fly Inductive Charging
}

DOI:

10.1007/s10846-017-0673-8

\section{Document Version}

Accepted author manuscript

Link to publication record in Manchester Research Explorer

\section{Citation for published version (APA):}

Arvin, F., Watson, S., Emre Turgut, A., Espinosa Mendoza, J. L., Krajnik, T., \& Lennox, B. (2017). Perpetual Robot Swarm: Long-Term Autonomy of Mobile Robots Using On-the-fly Inductive Charging. Journal of Intelligent \& Robotic Systems. https://doi.org/10.1007/s10846-017-0673-8

\section{Published in:}

Journal of Intelligent \& Robotic Systems

\section{Citing this paper}

Please note that where the full-text provided on Manchester Research Explorer is the Author Accepted Manuscript or Proof version this may differ from the final Published version. If citing, it is advised that you check and use the publisher's definitive version.

\section{General rights}

Copyright and moral rights for the publications made accessible in the Research Explorer are retained by the authors and/or other copyright owners and it is a condition of accessing publications that users recognise and abide by the legal requirements associated with these rights.

\section{Takedown policy}

If you believe that this document breaches copyright please refer to the University of Manchester's Takedown Procedures [http://man.ac.uk/04Y6Bo] or contact uml.scholarlycommunications@manchester.ac.uk providing relevant details, so we can investigate your claim.

\section{OPEN ACCESS}




\title{
Perpetual Robot Swarm: Long-term Autonomy of Mobile Robots Using On-the-fly Inductive Charging
}

\author{
Farshad Arvin · Simon Watson - Ali Emre Turgut · Jose Espinosa • \\ Tomáš Krajník · Barry Lennox
}

\begin{abstract}
Swarm robotics studies the intelligent collective behaviour emerging from long-term interactions of large number of simple robots. However, maintaining a large number of robots operational for long time periods requires significant battery capacity, which is an issue for small robots. Therefore, re-charging systems such as automated battery-swapping stations have been implemented. These systems require that the robots interrupt, albeit shortly, their activity, which influences the swarm behaviour. In this paper, a low-cost on-thefly wireless charging system, composed of several charging cells, is proposed for use in swarm robotic research studies. To determine the system's ability to support perpetual swarm operation, a probabilistic model that takes into account the swarm size, robot behaviour and charging area configuration, is outlined. Based on the model, a prototype system with 12 charging cells and a small mobile robot, Mona, was developed. A series of long-term experiments with different arenas and behavioural configurations indicated the model's accuracy and demonstrated the system's ability to support perpetual operation of multi-robotic system.
\end{abstract}

Keywords Swarm Robotics - Wireless Charging · Long-term Autonomy · Perpetual Swarm

F. Arvin, S. Watson, J. Espinosa, and B. Lennox

School of Electrical and Electronic Engineering, University of Manchester, Manchester, M13 9PL, United Kingdom E-mail: farshad.arvin@manchester.ac.uk

A. E. Turgut

Mechanical Engineering Department at Middle East Technical University, 06800 Ankara, Turkey

T. Krajník

Artificial Intelligence Centre, Faculty of Electrical Engineering, Czech Technical University, Prague, Czechia

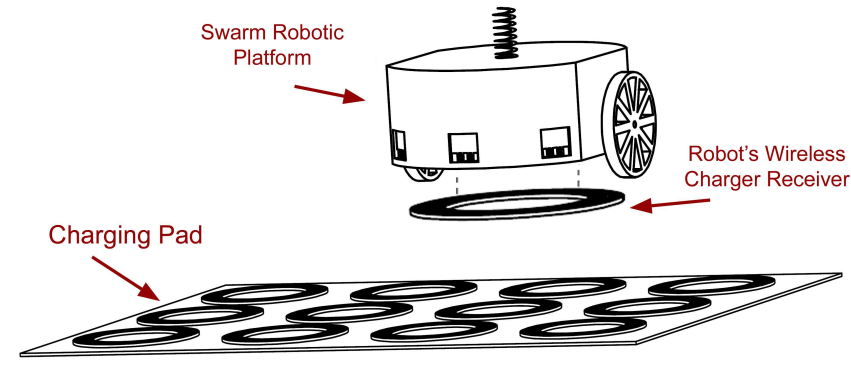

Fig. 1 Structure of the proposed system including: i) a mobile robot, Mona, ii) a wireless charger receiver attached to the robot, and iii) a charging pad including independent charging cells.

\section{Introduction}

Mobile robots are employed with growing frequency in many different fields such as exploration, education and domestic use (service and entertainment). Whilst robots could become our assistants and make our lives easier, their capability of reliable and safe long-term autonomous operation is still limited, which sometimes causes them to be considered a burden rather than a benefit $[1,2]$. One of the fundamental limitations is that experimental robotic platforms cannot operate for long time because of their limited battery capacity, and therefore, experiments that verify reliability of robotic methods in long-term scenarios are not common.

The scope of this work is concerned with the power management aspect of robotic swarms which are supposed to operate for arbitrarily-long periods of time. Mobile, untethered robots must carry an onboard power supply which needs to either be replaced or recharged when it has been depleted. Whilst the computation capabilities of small and medium sized mobile robots has increased significantly over the last decade, battery capacity did not follow Moore's law. 
This severely limits the use of small robots in most application areas to short missions ( $<1$ hour).

Swarm robotics is one of the promising approaches for mobile robot coordination, which takes inspiration from social insects seen in nature. In swarm robotics [3], local interactions among a group of relatively simple mobile robots, running a simple algorithm, result in a flexible, collective problem solving capability as seen in ants, bees and termite colonies. As highlighted in the seminal work by Şahin [4], one of the main criteria of swarm robotics is having a "large number of robots", typically at least 10-20. The number of robots being used in swarm robotics research studies increased significantly with swarm sizes now reaching up to 1000 robots [5]. Such a large number of robots itself presents a significant power management challenge. Furthermore, the emergence of some swarm-intelligent behaviours might require time which far exceeds the time of operation limited by the battery capacity.

In robotics, in order to tackle the power problem, several different approaches have been employed to date. The simplest, but most tedious way is to manually connect robots with low battery levels to chargers [6], or to (again manually) replace their batteries [7]. These approaches become cumbersome and inefficient when there is a large number of robots or when the experiment requires long time. In more advanced approaches, the robots seek charging stations by themselves when their battery level drops below a critical value [8] or they schedule their charging times in accordance with anticipated users' demands [9]. This still causes the robots to spend a significant fraction of their operation time on the charging station. Slow recharging can be solved by automated battery swapping systems [10], but even here the robot has to interrupt its current activity and visit the battery-swapping station.

To avoid the battery problem completely, one can supply the robots in a continuous manner. While single robots can be tethered and connected to energy supply directly [11], a tethered multi-robot system would be hard to manage, as the cables would get entangled over time. Another approach is a powered ground [1216], where the robots collect electricity continuously via direct contacts that are in touch with the ground they move on. These systems were successfully used in swarm experiments lasting several hours. However, the mechanical connectors get worn out and dirty over time, which affects the energy flow to the robots and that can significantly impact the behaviour of the entire swarm. This effect needs to be avoided in swarm experiments which are not concerned with energy autonomy. Moreover, these methods would be hard to combine with other systems where the ground is used for other purposes, e.g. the simulated pheromone [17]. Finally, researches [18-20] suggest to use wireless power transfer which does not suffer from the wear-and-tear of contact-based systems.

In this paper, a novel on-the-fly charging for robotic swarms is proposed. The system uses inductive (wireless) energy transfer to continuously keep the battery of each robot charged. Unlike other wireless systems, the proposed one consists of several charging pads, which allows to scale up its size simply by adding more of them, see Fig. 1. Furthermore, multiple pad configuration ensures homogeneous density of power and prevents interruption of the swarm operation even in case of charger failure. The system improves the state-of-the-art in: (1) Seamless operation; robots are not interrupted by charging, (2) Continuous charging despite of charging system and robot wear-and-tear, (3) Scalability; the system does not impose constraints on the number of robots or arena size, (4) Reliability; readily available commercial technology make the system reliable, and (5) Low cost; off-the-shelf components make the system inexpensive to build and operate.

The remainder of this paper is organised as follows: Section 2 provides a review of the existing body of work in terms of existing swarm robot platforms and their related charging systems. Section 3 provides an introduction to wireless inductive charging whilst Section 4 presents the realisation of the proposed system with different experimental configurations in Section 5. In Section 6, a probabilistic model of the charging scenarios is introduced and in Section 7, the results of the experiments are presented. Sections 8 and 9 present the discussions and conclusions of the work.

\section{Related Work}

A review of the most common swarm robotic systems is presented in this section with a specific focus on their power management capabilities and autonomy times.

Several mobile robot platforms exist for swarm robotic applications (see Table 1). Alice [21], a very small-sized platform, has been employed in many different swarm projects. The first design of Alice used two watch batteries, but solar panels and lithium batteries were employed later to increase the autonomy time [30]. AMiR [22] and Colias [23] are low-cost open-hardware platforms for swarm robotics research. They have 1-3 h of autonomy time depending on the tasks they are required to perform. Their batteries are charged manually by connecting to a charger. $E$ puck [24] is one of the most successful robots primarily designed for education. Due to its price and simplicity, 
Table 1 Comparison of size, autonomy time and charging method for some swarm robotic platforms

\begin{tabular}{lr|r|r|c} 
Robot & Ref & $\begin{array}{r}\text { Size } \\
{[\mathrm{cm}]}\end{array}$ & $\begin{array}{r}\text { Time } \\
\text { hours }]\end{array}$ & $\begin{array}{c}\text { Charging } \\
\text { method }\end{array}$ \\
\hline Alice & {$[21]$} & 2.2 & 10 & Manual/Solar \\
AMiR & {$[22]$} & 6.5 & 2 & Manual \\
Colias & {$[23]$} & 4.0 & $1-3$ & Manual \\
Droplet & {$[15]$} & 4.4 & - & Powered ground \\
E-puck & {$[24]$} & 7.5 & $2-4$ & Manual \\
Foot-bot & {$[25]$} & 13.0 & $1-3$ & Autonomous \\
Jasmine & {$[26]$} & 3.0 & $1-2$ & Autonomous \\
Khepera & {$[27]$} & 5.5 & $1 / 2$ & Autonomous \\
Kilobot & {$[28]$} & 3.3 & $3-24$ & Manual \\
Kobot & {$[7]$} & 12.0 & 10 & Manual \\
S-bot & {$[29]$} & 12.0 & 2 & Manual \\
SwarmBot & {$[8]$} & 12.7 & 3 & Autonomous
\end{tabular}

it is frequently employed in swarm robotics research. It has user-replaceable batteries and an autonomy time of 2-4 h. Foot-bot (a version of MarXbot platform [10]) was designed for swarm robotics research, specifically the Swarmanoid project [25] and it remains one of the most capable swarm platforms available. It has an autonomy time of 1-3 $\mathrm{h}$ depending on the configuration. Its battery can be changed both manually or automatically by a battery swapping station. Jasmine [26] is a small size robotic platform designed for implementation of bio-inspired swarm scenarios [31]. It has infra-red (IR) sensors both for proximity sensing and communication and an autonomy time of 1-2 h. Khepera [27] is one of the earliest modular robots designed for swarm robotics. While having small size, it has communication, stereovision and object manipulation capabilities. It has an autonomy time of $30 \mathrm{~min}$ and can be recharged both manually or through a docking station. Kilobot [28] is a relatively recent swarm robotic platform with novel functions such as group charging and group programming. Due to its simplicity and low power consumption, it has a long autonomy time of up to $24 \mathrm{~h}$. Robots are charged manually in large groups. Kobot [7] was specifically designed for swarm robotic research. Its sensoric equipment makes it an ideal platform for various swarm robotic scenarios such as coordinated motion. It has approximately $10 \mathrm{~h}$ of autonomy time, and its replaceable battery is recharged manually. $S$ bot [29] is one of the most influential and capable swarm robotic platforms ever built. Each s-bot has a unique gripper design capable of grasping objects and other sbots. They have an autonomy time of approximately $1 \mathrm{~h}$. It does not have replaceable batteries and it is charged manually. SwarmBot [8] is another swarm robotic platform with approximately $3 \mathrm{~h}$ of autonomy time and the ability to find and dock to charging stations which are placed on walls.
In autonomous charging, a charging station is used, which a robot should locate and dock to when its battery level is low. One of the earliest attempts in autonomous charging relied on a light source attached to the recharging station and light following behaviour of the robot [32]. Another autonomous recharging system used an environment map with known charging station location [33]. Later methods use IR [34] or vision $[35,36]$ to localise the recharging station. In [37], the use of a mobile charger robot, based on [38] was proposed. The mobile recharger was larger than the swarm robots and it could charge six robots simultaneously.

In the powered ground method, robots with conductive brushes move on a special floor with powered strips, e.g. Krieger et al. [14] used this approach to demonstrate ant-inspired foraging behaviour in several 30 minutes trial or Winfield and Nembrini [13] used a 9-meter wide powered floor to investigate swarm coherence. In Martinoli et al. [39] reported that their powered floor had $95 \%$ efficiency due to contact and frictional losses. Watson et al. [12] designed a similar powered ground system for embodied evolution experiments, which lasted over 3 hours. In the inductive charging method, there is a primary coil (transmitter) on the ground and a secondary coil (receiver) on the robot. Changing the magnetic field on the transmitter induces current on the receiver, which powers the robot. An inductive charging system with a single transmitter coil, that allowed perpetual, battery-less operation of 5 robots, was presented in [18]. Kepelson et al.[19] designed an inductive charging system with one primary transmitting loop and several relay loops to increase the charging coverage.

Except for a few platforms (Alice, Kilobot, and Kobot) most of the robots have an autonomy time around 1-3 h, which imposes a serious limitation for many swarm robotics scenarios. Some charging methods (tethering, manual charge) are not suitable for swarm experiments because they do not scale well with the number of robots. Other methods affect the swarm behaviour in short-term perspective by requiring that the robots interrupt their operation to recharge or swap their batteries. Finally, the contact-based powered ground methods are subject to wear-and-tear, which, in long-term, affects the energy transfer rate to the swarm and hence the swarm behaviour.

The most feasible alternative is based on wireless energy transfer. However, the systems presented in $[18$, 19] used a single transmitter, which does not provide the same magnetic flux across the entire arena, which can result not only in inefficient energy transfer but also can affect the swarm behaviour in undesired ways. 

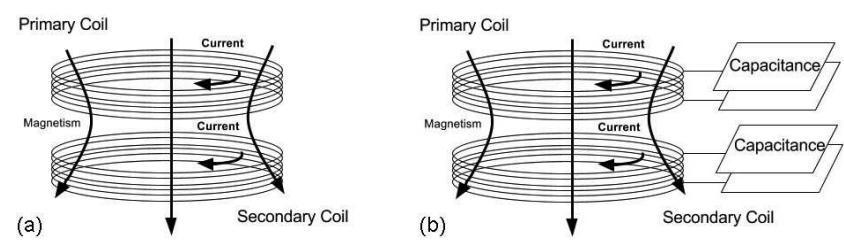

Fig. 2 (a) Tight coupled system (inductive coupled) and (b)Loose coupling system (magnetic resonance coupling) [41].

The solution proposed in this paper uses an inductive charging system which ensures homogeneous power distribution across the operational area of the swarm. The system is both scalable and tested for swarm applications and it was shown to be able to support continuous swarm operation for several days. In theory, the system could operate indefinitely. Unlike [18], the proposed system is scalable hence suitable for robot swarms and unlike [19], our system is more energy efficient and enables continuous operation of more numerous swarms in larger areas.

\section{Wireless Charging}

Wireless charging technology can be classified based on its working principle. Common methods include electromagnetic radiation charging, electric field coupling charging, and magnetic field coupling charging [40]. For short distance power transfer $(\sim \mathrm{cm})$, magnetic field coupling in the form of electromagnetic induction (inductive charging) is the primary technology in use.

\subsection{Inductive Charging}

Magnetic field coupling works by creating an alternating magnetic field, flux, in a transmitter (primary) inductor coil and converting the flux in a receiving inductor coil (secondary). Depending on the distance and alignment between the primary and secondary coils, inductive charging can be classified as either tight coupling or loose coupling. A model of how the system works is shown in Fig. 2.

\subsection{Tight Coupled Systems}

Tight coupled systems, or inductive coupling, operate when the primary coil generates a varying magnetic field across a secondary coil. The coils cannot be far from each other, as the near-field power attenuates to the cube of the distance between the two coils [42]. For more efficient operation, the secondary coil is tuned to

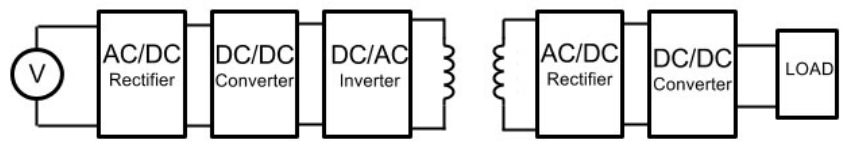

Fig. 3 Block diagram of a general wireless charging system

the operating frequency, which is normally in the $\mathrm{kHz}$ range.

These systems have a higher efficiency when transmitting power, however the distance between coils should be less than the diameter of the coils [41]. These type of systems are effective from a few millimetres to a few centimetres, however they are susceptible to misalignment.

An important consideration is that two tightly coupled coils cannot resonate at the same time. This means that there is a design trade-off for inductive charging systems between more efficiency or better performance when the coils are not aligned properly.

\subsection{Loose Coupled Systems}

Loose coupled systems are based on the principle of magnetic resonance coupling, where the secondary coil is part of a resonant circuit, which is tuned to the primary coil frequency. High energy transfer can be achieved over longer distances than inductive coupling [41], and one transmitter can transmit to multiple resonators [43]. These systems achieve a reasonable efficiency even when the coils are misaligned or not in the line of sight. A drawback is that they are more complex to implement than inductive coupling. The operating frequency is in the $\mathrm{MHz}$ range.

\subsection{Implementation of Inductive Charging}

A general block diagram of an inductive charging system is shown in Fig. 3. The transmitter is formed by an $\mathrm{AC} / \mathrm{DC}$ rectifier, which is connected to a mains power supply, a DC/DC converter to change the level of voltage and a $\mathrm{DC} / \mathrm{AC}$ inverter to make the varying magnetic field on the transmitter coil.

The receiver system consists of a receiving coil connected to an $\mathrm{AC} / \mathrm{DC}$ rectifier to create a $\mathrm{DC}$ voltage, then a DC/DC converter connected to the system load.

Tight coupled systems are generally composed of a single transmitting coil and a single receiving coil. Loose coupled systems can exist in complex configurations that contain up to four coils with impedance matching, relay resonator, and domino resonator systems $[44,45]$. 


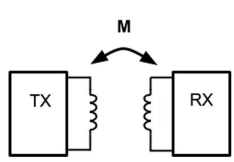

(a)

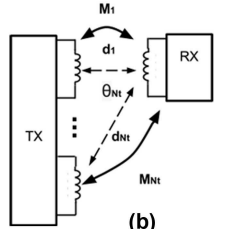

(b)

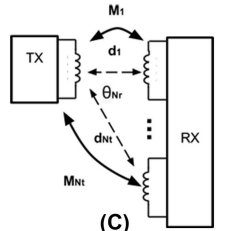

(C)
Fig. 4 Propagation models: (a) SISO, (b) MISO, and (c) MIMO.

\subsection{Propagation Models}

There are three fundamental near-field magnetic propagation models: single-input-single-output (SISO), multiple-input-single-output (MISO) and multi-input multi-output (MIMO) configurations. These configurations are shown in Fig. 4.

\subsubsection{SISO}

The transmission efficiency is highly dependant on the mutual inductance between the two coils, the quality factor $Q$, and the load matching factor. The quality factor indicates the energy loss during power transmission; the bigger $Q$ is, the less energy is lost. The load matching factor measures how tight the resonance frequencies are matched. The power received at the load of the receiver can be obtained with Eq. 1:

$P_{r}=P_{t} Q_{t} Q_{r} \eta_{t} \eta_{r} k^{2}$,

where $P_{r}$ is the received power, $P_{t}$ is the transmitted power, $\eta_{t}$ and $\eta_{r}$ are the efficiencies of the transmitter and receiver, $Q_{t}$ and $Q_{r}$ are the quality factors of the transmitter and receiver, and $k$ is the coupling coefficient that is determined by the coil alignment, distance, ratio of diameters, and shape of the coils.

\subsubsection{MISO}

In MISO systems each coil of a charger is coupled, at a resonant frequency, with a receiver. The power delivered to each receiver can be determined from Eq. 2: $P_{r}^{n}=P_{t}^{n} Q_{t}^{n} Q_{r} \eta_{t}^{n} \eta_{r} k_{n}^{2}\left(d_{n}\right)$,

where $N_{t}$ represents the number of transmitting coils, $P_{t}^{n}, Q_{t}^{n}$ and $\eta_{t}^{n}$ denote the transmitted power, quality factor and efficiency of the coil $n$ and $d_{n}$ denotes the distance between the charger coil $n$ and the receiver.

\subsubsection{MIMO}

In the MIMO transmission model, a receiver receives the power from each individual transmission coil separately. The receiver power at the load is given by Eq. 3:

$P_{r}^{n, m}=P_{t}^{n} Q_{t}^{n} Q_{r}^{m} \eta_{t}^{n} \eta_{r} k_{n, m}^{2}\left(d_{n, m}\right)$

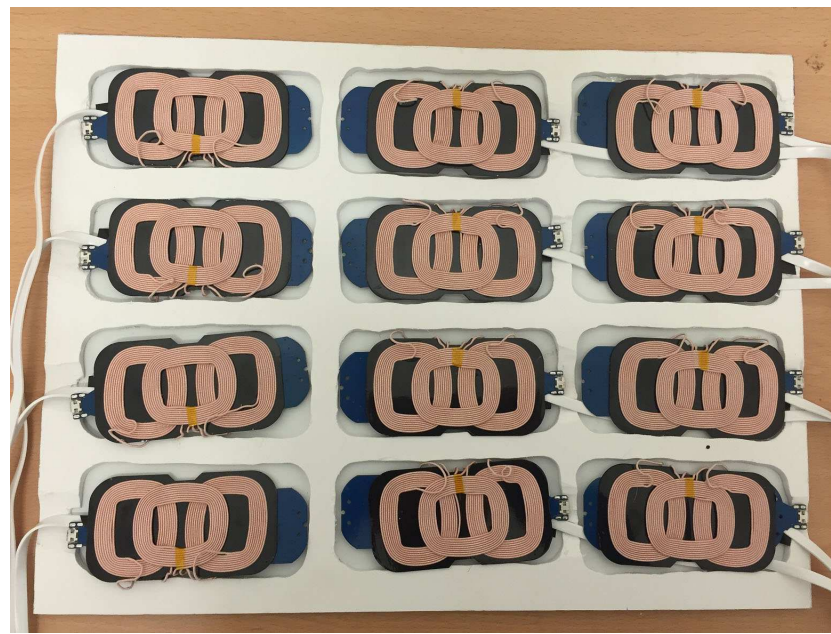

Fig. 5 The utilised prototype charging pad $\left(30 \times 40 \mathrm{~cm}^{2}\right)$ including 12 independent cells $\left(M_{4,3}\right)$ supplied with a USB hub $(5 \mathrm{~V}, 1 \mathrm{~A})$.

\section{System Implementation}

This section presents the design of the prototype system in three parts: i) the design of the charging pad and the robot platform, ii) the design of experiments to investigate the feasibility of the proposed system and iii) the behavioural improvement to increase the performance of the system.

\subsection{Arena Configuration}

The arena is a charging pad with a matrix of independent charging cells. In the developed prototype for this work, 12 cells were utilised as shown in Fig. 5.

The charging pad is represented by a matrix $M_{m, n}$ :

$$
M_{m, n}=\begin{array}{cccc}
c_{1,1} & c_{1,2} & \cdots & c_{1, n} \\
c_{2,1} & c_{2,2} & \cdots & c_{2, n} \\
\vdots & \vdots & \ddots & \vdots \\
c_{m, 1} & c_{m, 2} & \cdots & c_{m, n}
\end{array}
$$

where $c_{n, m}$ is a charging cell at position $(m, n)$ in the arena. The cells have a binary state; on or off ( 1 or 0$)$. In this work the size of the cell matrix is $M_{4,3}$.

Each cell is able to be activated or deactivated independently and can provide a maximum charging current of $1 \mathrm{~A}$. However, this is limited to $500 \mathrm{~mA}$ to prevent overheating. Fig. 6 shows (a) a charging cell and (b) a receiver module.

The method of charging selected for this work is a Tight Coupled SISO system, which deals with the misalignment issues described in Section 3.2 by using an RF communication to only provide power to the transmission coil when the receiver is in the 

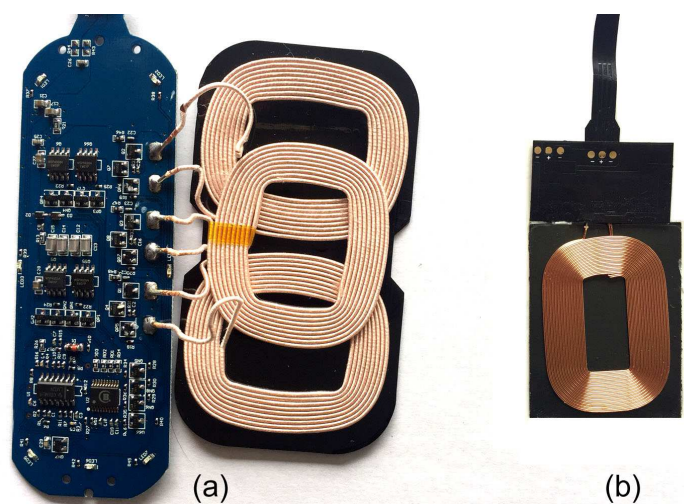

(b)

Fig. 6 (a) A charging cell with the extended active area and (b) a receiver antenna that is attached to the bottom layer of robot's board.

correct location. This reduces the power consumption and increases safety, as the transmission coils are only turned on when required. The wireless power transmitter and receiver circuits that were used in this work have been developed based on the typical application circuit proposed on the datasheets of the modules (BQ500210 and BQ51013B). The schematics of the circuits are presented in Appendix I.

The results of the preliminary experiments showed that there was a delay caused by the time taken for the receiver to connect to the transmitter, $\left(t_{c}\right)$, which is about $1 \pm 0.5 \mathrm{sec}$. This delay is a constant predefined time which the charger's and receiver's processors require to establish a connection and to avoid noise, hence at this stage of the research, there was no method available to eliminate this delay. The utilised charging cells are low-cost (£4 per one) off-the-shelf modules which can be easily added to extend size of the arena.

\subsection{Robot Platform}

To investigate the feasibility of the proposed system, a mobile robot, called Mona, was developed. The robot is a customised design of a previously developed robot, Colias [23]. It is specially designed to utilise an inductive charging approach as well as several additional functions such as a radio frequency (RF) transceiver and battery level monitoring module. It is a low-cost $(£ 30)$ and small yet capable robot with a diameter of approximately $7 \mathrm{~cm}$.

Fig 7 shows a Mona robot and its modules. The robot has been designed as a modular platform allowing deployment of additional modules that are attached on top of the platform, such as a vision board [46]. The upper board could be used for highlevel tasks such as inter-robot communication and user programmed scenarios for swarm robotic applications.

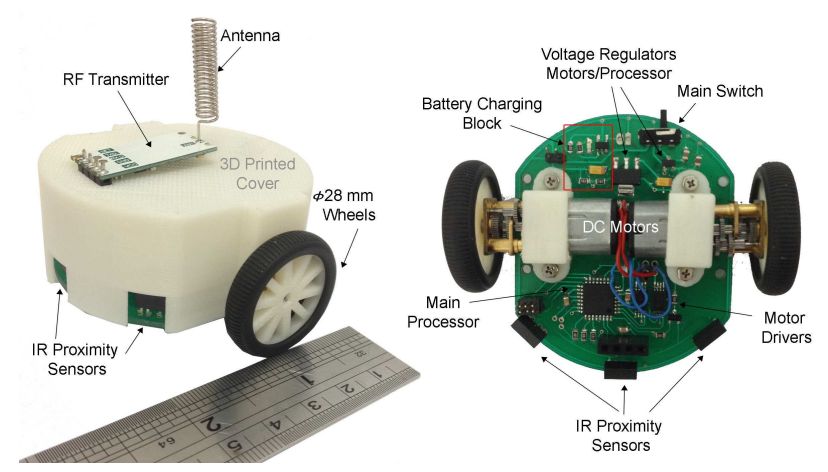

Fig. 7 Mona Robot with a 3D printed case. It has a very limited sensing ability including 3 IR proximity sensors, $\mathrm{RF}$ transmitter module to send battery level, a Li-Po battery charging circuit, two voltage regulators for motor and main processing unit, two $29 \mathrm{~mm}$ diameter wheels, DC motor drivers (H-bridge), two gearhead DC motors, and a temperature sensor at the bottom of the robot to monitor the ambient temperature.

The platform board is designed for low-level functions such as a power management, motion control, and communication between the robot and the charging cells.

An AVR 8-bit microcontroller (ATMEGA-168PA, with $16 \mathrm{~KB}$ in-system self-programmable flash memory and $1 \mathrm{~KB}$ internal SRAM) is utilised as the main processor. Two micro DC gearhead motors (with a high gear ratio of 400:1) and two wheels with diameter of $29 \mathrm{~mm}$ move Mona with a maximum speed of $15 \mathrm{~mm} / \mathrm{s}$. The arena's surface is made of methyl methacrylate (Perspex) which ensures no slippage of the robot wheels with a rubber tire. Based on results of preliminary experiments on the effective distance between transmitter and receiver coils, the size of wheels were chosen $29 \mathrm{~mm}$. This size allows to keep the transmitter-receiver distance below $10 \mathrm{~mm}$, which maintains the efficiency of the power transmission. The receiver we attached to the robots is relatively light (approx. $5 \mathrm{~g}$ ) and hence robots behave as without an additional payload. The rotational speed for each motor is controlled individually using a pulsewidth modulation (PWM) approach which is explained in [47]. Each motor is driven separately by an H-bridge DC motor driver, and consumes between $100 \mathrm{~mW}$ and $200 \mathrm{~mW}$ of power, depending on the load and speed.

Mona employs three short-range IR proximity sensors in front to detect and avoid obstacles within a distance of approximately $2 \pm 0.5 \mathrm{~cm}$ [48]. Since Mona uses basic IR proximity sensors without encoders or filters, it is not possible to deploy it outdoors. Power consumption of the robot with full forward motion with all devices on (in an uncluttered arena with only walls) is about $700 \mathrm{~mW}$, but with LEDs off and with 
Table 2 Power consumption of Mona's modules at $3.7 \mathrm{~V}$

\begin{tabular}{l|r|r} 
Module & Mode & Power $[\mathrm{mW}]$ \\
\hline Motors & $4 \mathrm{~mm} / \mathrm{s}$ & 225 \\
Motors & $12 \mathrm{~mm} / \mathrm{s}$ & 340 \\
IR Proximity & continuous & 190 \\
IR Proximity & $\sim 5 \mathrm{~Hz}$ & 50 \\
RF Tranceiver & transmitting & 70 \\
RF Tranceiver & idle & 30 \\
LEDs & - & 50 \\
Others & - & 50
\end{tabular}

optimising the IR proximity sensors and RF operation, the robot consumes about $450 \mathrm{~mW}$. A $3.7 \mathrm{~V}, 240 \mathrm{mAh}$ lithium battery is utilised as the main power source, which allows for approximately an hour of continuous operation. Table 2 illustrates the power consumption of Mona's modules.

The power consumption outlined in the Table 2 shows that the highest power consumption is for the motors, especially when the speed is high. The IR proximity sensors are the second highest consumer of power. However, with careful management through their occasional use only, the operational time of the robot can be increased.

To avoid transmitter magnetic field from interfering with Mona's processor, electromagnetic (EMI) shield is attached to the bottom of the main board in between receiver's coil and the robot's PCB. The shielding layer also isolates the receiver from magnetic field generated by the DC motors.

\section{Experimental Setup}

Three different sets of experiments were conducted: i) to test the feasibility of an inductive charging; a single transmitter in the pad and a single receiver attached to a robot, ii) to investigate the effects of different pad configurations on the performance of the system, and iii) to apply behavioural adjustments to the robots to provide a longer autonomy time and to achieve perpetual autonomy.

\subsection{Static Configuration Experiments}

\subsubsection{Hardware Feasibility}

A random walk scenario was performed at a speed of $10 \mathrm{~mm} / \mathrm{s}$ without any active charging. The results of the experiment provided a diagram of a long-term trajectory path of the robot showing how uniformly it covered the arena. The walking algorithm was a simple forward motion, with the robot turning to a random direction to avoid collisions with the walls.
A visual localisation system, developed in [49,36], was used to track the robots during the experiment using an overhead camera.

To demonstrate the amenability of the charging pad to be used in various swarm robotic scenarios, where the robot remains on a charging cell for a long period of time (e.g. as a food source, a nest, or a defined charging station [50-53]), an experiment was conducted to evaluate the pad's thermal profile. A Mona robot was placed, stationary, in the middle of a charging cell and the battery level and charging pad's temperature were recorded. During this experiment, the battery was charged from $3.4 \mathrm{~V}$ to $4.2 \mathrm{~V}$.

\subsubsection{Robot Speed}

The proposed system is a simultaneous charging approach which provides a small amount of power over a short span of time, during which the robot's receiver and arena transmitter are in resonance. The typical duration of the resonance strongly depends on the robot speed. To investigate the effects of the robot's speed on the performance of the system when all 12 chargers were activated. three sets of experiments were run, with the robot's forward speed set at $v_{o} \in\{4,8,12\} \mathrm{mm} / \mathrm{s}$. The duration of each experiment was $60 \mathrm{~min}$ and it was repeated for each configuration 5 times. The battery level was logged every 5 sec using an RF transmitter. For comparison, the experiment was run for each speed with the charging pads deactivated.

\subsubsection{Number of Cells}

To investigate the effects of the number of charging pads on autonomy time of Mona, a set of experiments were conducted where different numbers of cells $\left(n_{c} \in\right.$ $\{4,8,12\})$ at random locations were activated. The robot moved with a minimum defined speed of $4 \mathrm{~mm} / \mathrm{s}$. The experiment was repeated for each configuration 5 times with activated chargers in random positions for a duration of $60 \mathrm{~min}$. The battery level was logged every $5 \mathrm{sec}$.

\subsubsection{Cell Topology}

In this setup, different numbers of chargers with different arrangements in the arena were activated. Three different cell arrangements were tested (see Fig. 8): i) six chargers along walls (CC-a), ii) six chargers at the centre and sides (CC-b), and iii) four chargers at the corners of arena (CC-c). The robot moved at minimum speed of $4 \mathrm{~mm} / \mathrm{s}$ and its battery level was recorded during $60 \mathrm{~min}$ of experiment. Each experiment was repeated 5 times. 

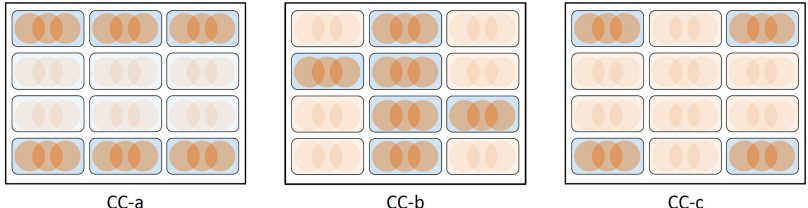

Fig. 8 Different cell configurations (CC-a: six chargers at top and bottom lines near the walls are activated, CC-b: six charger in centre line and sides are activated, and CC-c: four chargers at the corners are activated).

\subsection{Behavioural Performance Improvement}

These experiments investigated the effects of the robot charging behaviour on its ability of perpetual operation.

The aim was to define the robot's behavioural functions to improve the performance of the system. Here, a basic behaviour was proposed, which did not affect the robot's main task, yet provided a longer charging time. The behaviour was to reduce the speed of the robot to $v_{c}$ when detecting a charging cell. This would prolong the total time a robot stays on an active area of a charging cell, resulting in harvesting more energy. A similar modification of the behaviour of individuals - dynamic velocity - to increase the performance of an aggregation scenario in a swarm robotic system was proposed in [54].

In this scenario, a robot moved at a speed of $v_{o}=8$ $\mathrm{mm} / \mathrm{s}$ and reduced its speed down to $v_{c}=3 \mathrm{~mm} / \mathrm{s}$ when detecting a charging cell. To investigate the performance of the proposed behavioural modification, two sets of experiments with different numbers of chargers were conducted $\left(n_{c} \in\{6,12\}\right)$. In the case of 6 active chargers, cells were randomly chosen for each run. The battery level was logged during $60 \mathrm{~min}$ of experiment.

Another behavioural adjustment was to use IR emitters only occasionally. Mona could scan proximity sensors with a very low frequency (e.g. $<5 \mathrm{~Hz}$ ) to reduce the power consumption. As shown in Table 2, this adjustment significantly reduces the power consumption.

\subsection{Marathon Walk}

For this experiment, all 12 chargers were activated and a robot was deployed to perform a marathon walk so that the performance of the proposed charging pad in a long-term random walk scenario over 12 hours could be investigated. The robot utilised the behavioural improvements (dynamic velocity) which were tested in Section 5.2. The proximity sensors were operated with a frequency of $3 \mathrm{~Hz}$.
The energy level of the battery was tracked during the 12 hour marathon walks with two different sets of speeds, $\left(v_{o}, v_{c}\right) \in\{(5,3),(8,4)\} \mathrm{mm} / \mathrm{s}$.

\subsection{Multi-robot Exercise}

In this set of experiments, three Mona robots were deployed with a similar random walk scenario as the marathon walk (see Section 5.3). No inter-robot interactions were defined (e.g. swarm robotic scenarios) to test the feasibility of the proposed system without getting the benefits from swarm interactions. The only impact on the behaviour of the robots would be the additional number of turns due to collision avoidance.

Samples of battery voltage were recorded every $10 \mathrm{sec}$ for each robot separately during $12 \mathrm{~h}$ of experiments.

\subsection{Metrics and Statistical Analysis}

The metric used to evaluate the performance of the charging system on the robot's autonomy was the energy of the battery. For the purpose of this work, the measured variable was the battery voltage, $E_{b}$. The autonomy time of the robot (its life span) was measured as the time taken for the battery voltage to drop below $3.4 \mathrm{~V}$.

The results of all of the experiments were statistically analysed. A multi-factor analysis of variance (ANOVA) and the F-test method [55] were used in the analysis. The Tukey Pairwise Comparisons were also used to find the most significant setting for the investigated configurations.

The standard values of the constants and variables, which were used in this study are listed in Table 3.

Table 3 Experimental values or range for variables and constants

\begin{tabular}{c|l|l} 
Values & Description & Range / Value(s) \\
\hline$n_{c}$ & Number of deployed chargers & $\{4,8,12\}$ \\
$n_{r}$ & Number of deployed robots & $\{1,3\}$ \\
$v_{o}$ & Robot forward velocity & $\{3,4,5,8,12\} \mathrm{mm} / \mathrm{s}$ \\
$v_{c}$ & Charging forward velocity & $\{3,4\} \mathrm{mm} / \mathrm{s}$ \\
$T_{a}$ & Autonomy time & $0-12$ hours \\
$t_{c}$ & Time of connection & $1 \pm 0.5 \mathrm{sec}$ \\
$t$ & Time & 0 to 12 hours
\end{tabular}

\section{Probabilistic Modelling}

Due to the stochastic characteristics of swarm scenarios, a probabilistic approach is the most appropriate 
method of modelling the behaviour of the robots. Several probabilistic models have been proposed in swarm robotics $[56,57]$. A macroscopic model of an aggregation behaviour was proposed by Soysal and Şahin [58], which predicted the final distribution of the system. Bayındır and Şahin [59] proposed a macroscopic model for a self-organised behaviour using probabilistic finite state automata, which modelled the behaviour of the swarm system. A Langevin equation to model the collective behaviour of a swarm was used by Hamann [60]. Schmickl et al. [61] proposed macroscopic modelling of an aggregation scenario using a Stock \& Flow model. In previous work [62], a power-law equation model to predict the behaviour of a swarm was proposed.

For the model in this study, it was assumed that the robot had a circular cross-section with a diameter $d_{r}$, the shape of the individual charging cells was rectangular with dimensions $x_{c}, y_{c}$ and that the arena was also rectangular with its length and width denoted by $x_{a}, y_{a}$.

Since a robot can move only inside of the arena walls, its centre can move only inside of a Minkowski difference between the arena and the robot shape, i.e. inside of a $\left(x_{a}-d_{r}\right) \times\left(y_{a}-d_{r}\right)$ rectangle. Similarly to that, a robot charges only if its charging coil and the charging cell overlap significantly - that is, the receiving coil centre is inside of the $\left(x_{c}-d_{c}\right) \times\left(y_{c}-\right.$ $d_{c}$ ) rectangle, where $d_{c} / 2$ corresponds to the minimal distance of the charging coil centre from the charging cell border. Moreover, coupling of the robot to the charging cell takes a finite time, denoted as the time of connection, $t_{c}$, and thus, a robot that enters a charging cell with speed $v_{o}$, will start to charge when its centre is already $v_{o} t_{c}+d_{c} / 2$ inside of the charging cell. Thus, assuming that a robot moves in a way that the probabilistic distribution of its position inside of the arena is uniform, the probability that it is charging is:

$p_{c}^{\prime}=n_{c} \frac{a_{c}}{a_{a}} \frac{v_{o}}{v_{c}}=\frac{n_{c} v_{o}\left(x_{c}-d_{c}-v_{o} \frac{t_{c}}{2}\right)\left(y_{c}-d_{c}-v_{o} \frac{t_{c}}{2}\right)}{v_{c}\left(x_{a}-d_{r}\right)\left(y_{a}-d_{r}\right)}$

where $n_{c}$ is the number of charging cells, $v_{c}$ is the robot speed when detecting the charging signal, $v_{o}$ is the robot operating speed and $a_{c}$ and $a_{a}$ are the effective areas of the arena and the charging cells respectively. Since a robot can operate perpetually only if its energy balance is non-negative, then

$p_{c}^{\prime} w_{c}-w_{o} \geq 0$,

where $w_{c}$ is the charging power and $w_{o}$ is the robot's power consumption during routine operation. The above equations consider only a single robot moving inside of an arena. In the case of a higher number of robots, the fact that the charging cells work in an exclusive way needs to be taken into account. In particular, if a robot enters the charging cell area, it will only charge if there is not another robot using the cell already. Thus, if there are $n_{r}$ robots on the arena, the probability that a robot charges is

$p_{c}=n_{c} \frac{a_{c}}{a_{a}} \frac{v_{o}}{v_{c}}\left(1-\frac{n_{r}-1}{n_{c}} p_{c}\right)$.

Expressing $p_{c}$ from (7) gives

$p_{c}=n_{c} \frac{a_{c} v_{o}}{a_{a} v_{c}+\left(n_{r}-1\right) a_{c} v_{o}}$,

which allows the probability to be calculated that a robot is charging on an arena with $n_{c}$ charging pads and $n_{r}$ robots.

Since a perpetual operation of the robot swarm requires that Eq. (6) is satisfied for every robot, combining Eq. (8) and (6) results in

$n_{c} \frac{w_{c}}{w_{o}} \frac{a_{c} v_{o}}{a_{a} v_{c}+\left(n_{r}-1\right) a_{c} v_{o}} \geq 1$,

which gives a relationship between the number of robots $n_{r}$, charging cells $n_{c}$, effective cell areas $a_{c}$, arena area $a_{c}$, charging power $w_{c}$, power consumption $w_{o}$ and robot operating speed $v_{o}$ and robot's speed when it is charging $v_{c}$.

Note, that if a charging cell lies close to the arena border, the probability of a robot standing on it is higher, because the robot has to turn in order to avoid the arena wall, which increases the probability of the robot being on such a 'border' cell. In this model, this effect is neglected, but it should be kept in mind that cells around arena borders are more likely to be occupied by robots. To determine how many charging cells are needed to be placed under an arena in order to support a swarm of $n_{r}$ robots, Eq. (9) needs to be rewritten as

$n_{c} \geq \frac{w_{o}}{w_{c}}\left(\frac{a_{a} v_{c}}{a_{c} v_{o}}+n_{r}-1\right)$.

\subsection{Current Arena with a Single Robot}

The arena used for these experiments was 400 $\times 300 \mathrm{~mm}^{2}$ and the robot diameter was $70 \mathrm{~mm}$. Measurements showed that the centre of the robot's coil had to be at least $10 \mathrm{~mm}$ inside the $80 \times 40 \mathrm{~mm}^{2}$ charging pad in order to start charging. Thus, if the operational speed of a robot is $8 \mathrm{~mm} / \mathrm{s}$ and the time of connection is $1 \mathrm{~s}$, then the effective area of the charger is $56 \times 16 \mathrm{~mm}^{2}$. Moreover, the power provided by the charging pad was about $2 \mathrm{~W}$ and the robot consumption was about $0.45 \mathrm{~W}$. Substituting these values into Eq. (9) gives

$12 \geq \frac{v_{c}}{v_{o}} \frac{450}{2000} \frac{330 \times 230}{56 \times 16}$, 


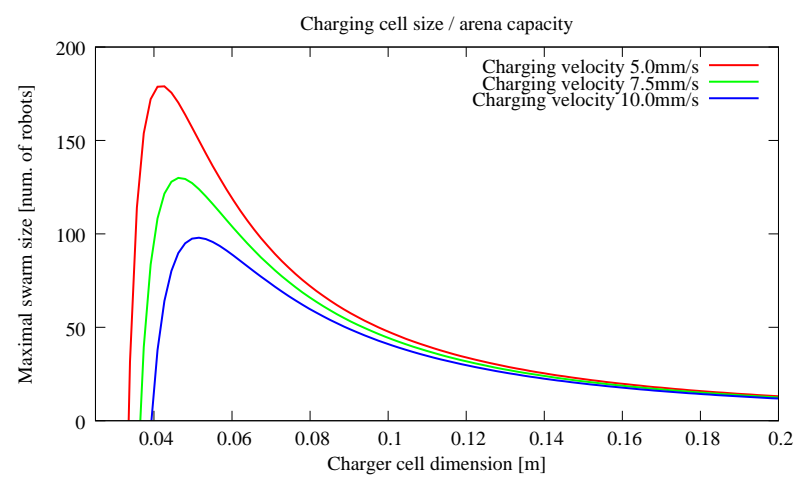

Fig. 9 Maximal swarm size vs charger cell dimensions. Nominal robot velocity is $10 \mathrm{~mm} / \mathrm{s}$. The graph shows that lowering robot velocity during charging allows to support larger swarms.

which gives a relationship between the charging and operational speed of a single robot:

$v_{o} \geq 1.59 v_{c}$,

which means that the robot has to slow down by $\sim 40 \%$ when detecting the charging station in order to stay perpetually operational. Note that in this case, the effective charging area $a_{c}$ is negligible compared to the size of the area $a_{a}$, and therefore, the probability of conflict with another robot $p_{f}$ would be about $3 \%$. This indicates that each additional robot requires that the charging speed is decreased only slightly (see the last part of Eq. (10)). In other words, setting the charging speed $v_{c}$ to $50 \%$ of the normal operational speed $v_{o}$ should allow operation of at least 10 robots.

\subsection{Supporting Larger Swarms}

To determine how many robots could be supported by a given configuration, Eq. (9) could again be rewritten as

$n_{r} \leq n_{c} \frac{w_{c}}{w_{o}}-\frac{a_{a} v_{c}}{a_{c} v_{o}}+1$

which suggests that the maximal swarm size $n_{r}$ increases linearly with the number of charging cells $n_{c}$.

\subsection{Optimal charger cell size}

The logical progression of the model is to examine what the maximal swarm size is that could be perpetually supported by an arena of given parameters. Assume that the size of the charging cells could be chosen and that they can be used to cover the entire arena without overlapping each other. If the assumption is made of a square arena with sides $x_{a}, y_{a}$ and square $x_{c} \times x_{c}$ charging cells, the question is what is the optimal size $x_{c}$ of the charging cells. A larger number of smaller cells decreases the competition for energy between the swarm robots by lowering the probability that two robots are located on the same charging cell. Conversely, in the extreme case, a single charging cell that covers the entire arena $x_{c}=x_{a}$ can support only one robot.

A larger number of smaller cells provide a smaller charging area than a lower number of larger cells because of the fact that a robot has to be inside of a cell completely in order to charge. Again, if the cell sizes are equal or smaller to the robot charging coil size, i.e. $x_{c}=d_{c}$, then the number of conflicts will be minimal, but the effective charging area of each cell will be zero. Assuming that the entire arena is covered with charging cells of a uniform size, i.e. the number of chargers $n_{c}$ is $\left(x_{a} / x_{c}\right)\left(y_{a} / x_{c}\right)$, then

$n_{r} \leq \frac{x_{a}}{x_{c}} \frac{y_{a}}{x_{c}} \frac{w_{c}}{w_{o}}-\frac{v_{c}}{v_{o}} \frac{\left(x_{a}-d_{r}\right)\left(y_{a}-d_{r}\right)}{\left(x_{c}-d_{c}-v_{o} \frac{t_{c}}{2}\right)^{2}}+1$.

For the estimated parameters of the system, the dependence of the swarm size on the charger size will look as shown in Fig. 9. As the model suggests, the optimal size of the charging pad for a $40 \times 30 \mathrm{~cm}^{2}$ arena is $5 \mathrm{~cm}$. Thus, the array of $8 \times 6$ chargers could theoretically provide energy for a swarm of almost 100 robots, which would roam with velocities of up to $10 \mathrm{~mm} / \mathrm{s}$.

\section{Experimental Results}

This section presents the results of the experiments outlined in Section 4.

\subsection{Hardware Feasibility Test}

\subsubsection{Trajectory and Coverage}

The first experiment was to check the moving behaviour of the robot. In this experiment, Mona moved with a speed of $10 \mathrm{~mm} / \mathrm{s}$ without any active charger. Fig. 10 illustrates a trajectory path of Mona during a one hour random walk. The tracking path showed that the robot uniformly explored the arena, hence the chosen random walk algorithm was a suitable scenario that passes all the charging cells.

\subsubsection{Heating Profile}

In this experiment the robot's temperature and battery voltage were recorded, and the temperature at a charging transmitter that was placed inside of the charging 


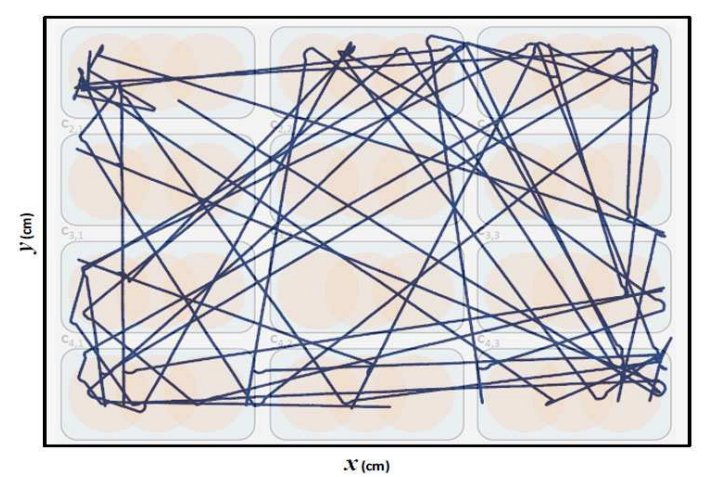

Fig. 10 Trajectory plan of Mona during 60 min random walk. The arena size is $30 \times 40 \mathrm{~cm}$.

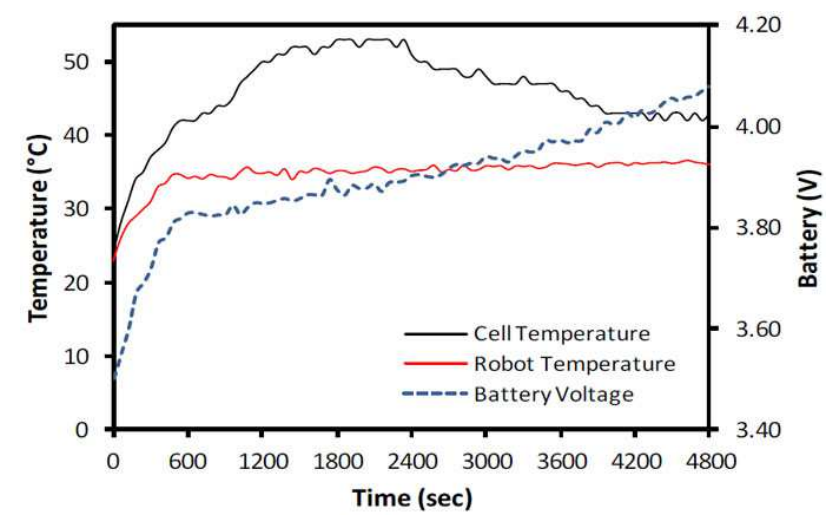

Fig. 11 Temperature profile of the robot receiver and the inside of the charging pad during $80 \mathrm{~min}$ of the battery recharging process.

pad. Fig. 11 shows two temperature profiles during 80 min of the recharging process of the robot's battery. The recorded data reveals that the temperature inside the pad increased to $52{ }^{\circ} \mathrm{C}$. Since the charging pad does not have any cooling channels, the temperature rise was expectable as a result of trapped hot air. However, the temperature change at the robot's receiver was not noticeable.

\subsection{Speed of Motion}

Fig. 12 illustrates the voltage level of the battery during 60 min experiments with a random walking robot at different speeds of $v_{o} \in\{12,8,4\} \mathrm{mm} / \mathrm{s}$. The results show that in all sets of experiments the reduction in battery level improved when the charging pad is activated. However, the median of the results from the robot at speeds of $4 \mathrm{~mm} / \mathrm{s}$ and $8 \mathrm{~mm} / \mathrm{s}$ showed a higher performance improvement in comparison to the robot with a speed of $12 \mathrm{~mm} / \mathrm{s}$. It can be seen that, as the probabilistic model in Section 6 suggests, the charging period relies on the motion speed of the robot. The results obtained when using different robot speeds illustrated that a low speed robot received a higher amount of energy than a faster robot due to the longer period its receiver overlapped with the transmitter cell. Therefore, reducing the speed of the robot increased its autonomy time.

The results of different sets of experiments were statistically analysed using two-way ANOVA. The time and speed of the robot were used as two independent factors and the battery voltage $\left(E_{b}\right)$ as the response. Table 4 shows the results of the statistical analysis. The results revealed that both factors - time and speed of robot - have a significant $(P \leq 0.05)$ impact on the energy harvesting of the robot.

Table 4 Statistical analysis of the results (ANOVA)

\begin{tabular}{l|c|c} 
Factors & $P$-value & $F$-value \\
\hline Time, $t$ & 0.000 & 3.952 \\
Speed, $v_{o}$ & 0.000 & 4.285
\end{tabular}

\subsection{Number of Charging Cells}

This set of experiments investigated the effects of the number of active chargers, $n_{c}$, on the performance of the system. Fig. 13 shows the obtained results from three different configurations, $n_{c} \in\{4,8,12\}$. The results revealed that an increase in the number of active chargers increased the performance of the system by harvesting more energy during the robot's random walk.

The results of statistical analysis with two factors time and number of active chargers - are illustrated in Table 5. The results showed that both factors impact the performance significantly $(P \leq 0.05)$.

Table 5 Statistical analysis of the results (ANOVA) with different number of active chargers

\begin{tabular}{l|c|c} 
Factors & $P$-value & $F$-value \\
\hline Time, $t$ & 0.000 & 7.524 \\
No. of Charger, $n_{c}$ & 0.000 & 24.628
\end{tabular}

\subsection{Charging Cells Arrangements}

This set of experiments investigated the effects of the different cell topologies on the performance of the energy harvesting. Fig. 14 illustrates the results from three different topologies (which are defined in Fig. 8). The results showed that the configuration CC-a has a higher amount of energy harvesting than the other two 


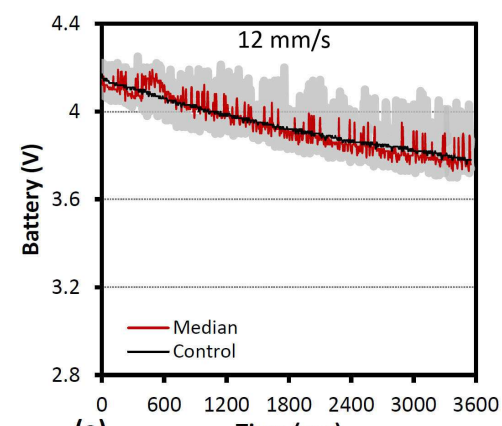

(a)

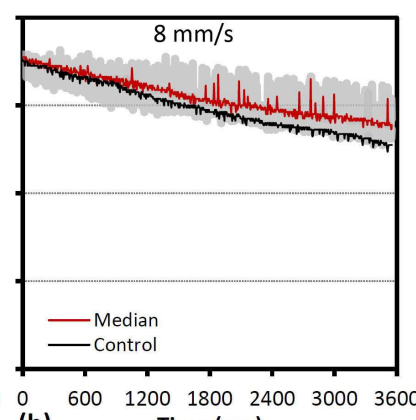

(b) Time (sec)

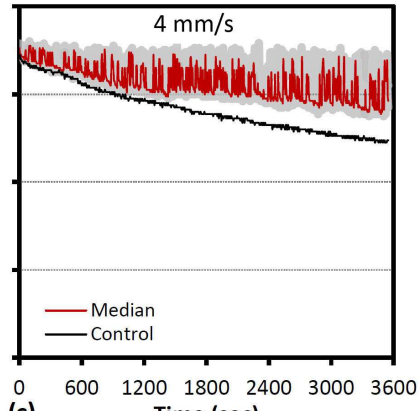

(c) Time (sec)

Fig. 12 Recorded battery voltage during $60 \mathrm{~min}$ of experiments with a forward speed of $v_{o} \in\{12,8,4\} \mathrm{mm} / \mathrm{s}$. The red line indicates the median of results with active chargers, the shaded area indicates range of results (between min and max) and the black line indicates the results of experiments without an active charger (control).

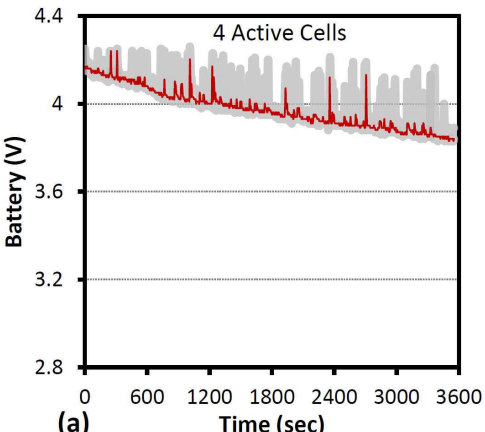

(a)

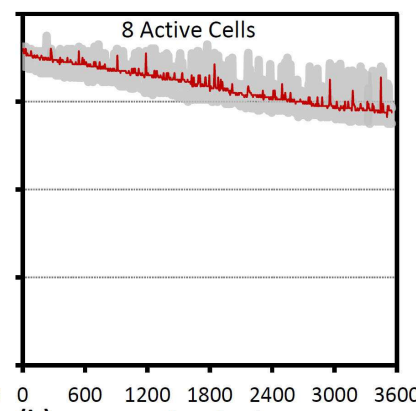

(b)

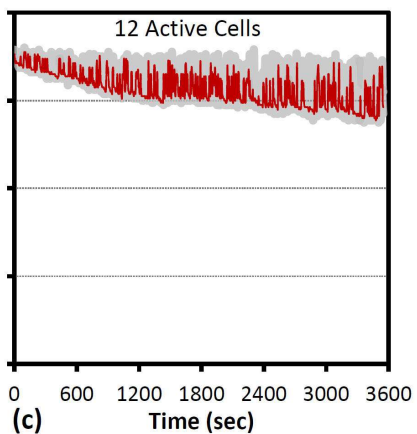

(c)

Fig. 13 Recorded battery voltage during $60 \mathrm{~min}$ of random walk at a speed of $4 \mathrm{~mm} / \mathrm{s}$ with different number of active chargers, $n_{c} \in\{4,8,12\}$. The red line indicates the median of results and the shaded area indicates range of results (between min and $\max )$.

settings. In the first cell configuration (CC-a), where six cells were activated along the top and bottom walls, Mona stayed longer on the chargers' area due to the turn-in-place trajectory of the obstacle avoidance. This resulted in harvesting higher amounts of energy than the other two scenarios.

The results were statistically analysed as shown in Table 6 . It can be seen that both factors (time and topology) significantly impact $(P \leq 0.05)$ the system.

Table 6 Statistical analysis of the results (ANOVA) with different configurations of active chargers

\begin{tabular}{l|r|r} 
Factors & $P$-value & $F$-value \\
\hline Time, $t$ & 0.000 & 9.861 \\
Topology & 0.004 & 5.704
\end{tabular}

\subsection{Behavioural Improvement}

This set of experiments improved the walking behaviour of the robot by using a dynamic velocity approach. As shown in the diagrams (see Fig. 15), the performance of the system improved significantly.
The results of the statistical analysis revealed that time, $t$, did not have a significant impact on the performance of the system ( $P$-value $=1$ and $P$ value $=0.574$ with 6 and 12 chargers, respectively). The results were also analysed using the multi-factor ANOVA method and the results are shown in Table 7. The most important result is to demonstrate that the reduction in battery energy of the robot does not depend on time $t$.

Table 7 Statistical analysis of the results (ANOVA) for improved walking behaviour

\begin{tabular}{l|r|r} 
Factors & $P$-value & $F$-value \\
\hline Time, $t$ & 0.980 & 0.582 \\
No. of Charger, $n_{c}$ & 0.686 & 0.897
\end{tabular}

\subsection{Marathon Walk}

The purpose of this experiment was to investigate the main proposal of a perpetual swarm. Mona walked nonstop for 12 hours and more than 8600 battery samples were recorded. Fig. 16 illustrates the recorded battery 


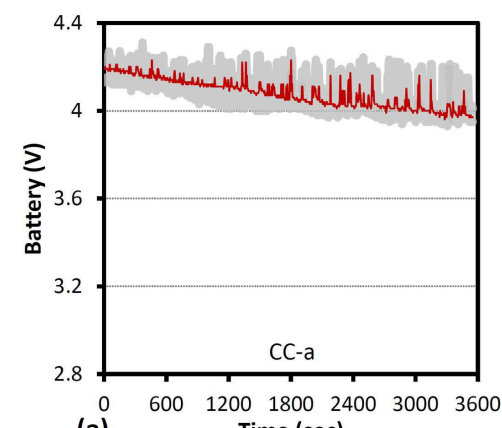

$\begin{array}{rrrrr}0 & 600 & 1200 & 1800 & 2400 \\ \text { (a) } & & \text { Time (sec) }\end{array}$

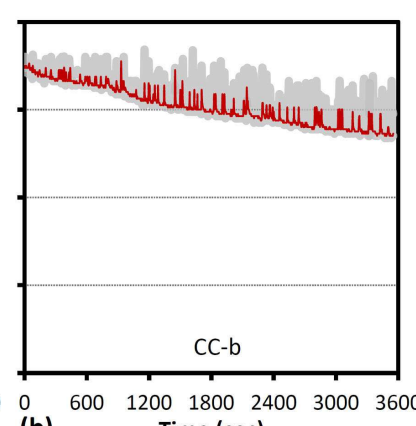

(b) Time (sec)

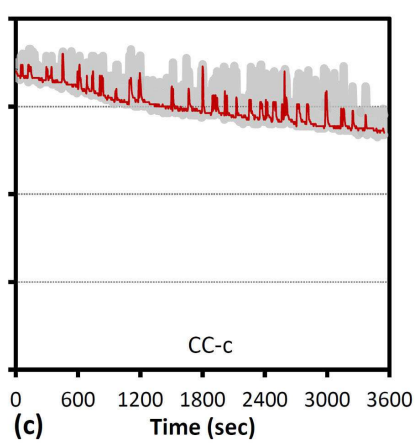

(c) Time (sec)

Fig. 14 Recorded battery voltage during $60 \mathrm{~min}$ of random walk at a speed of $4 \mathrm{~mm} / \mathrm{s}$ with different cell configurations (CC-a, CC-b and CC-c, as shown in Fig. 8). The red line indicates the median of results and the shaded area indicates range of results (between min and max).

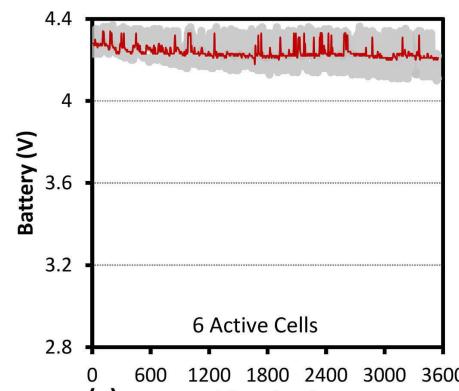

(a)

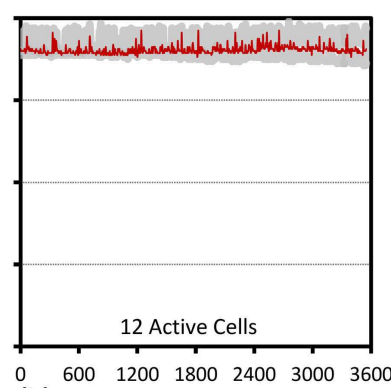

(b)
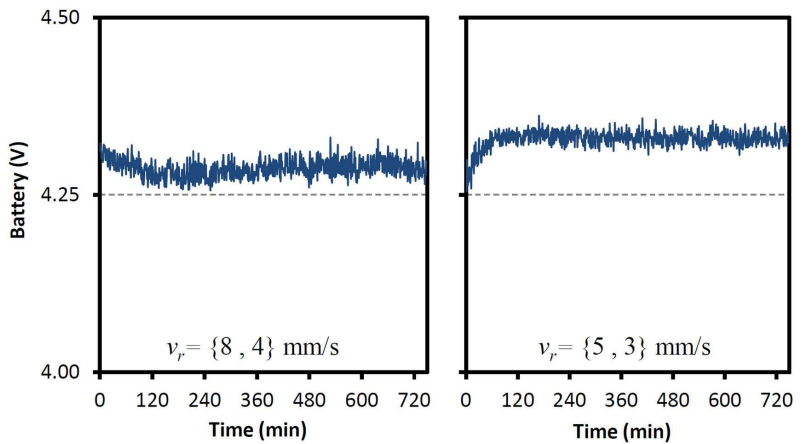

Fig. 16 Recorded battery voltage during 12 hours of random walk using an improved walking scenario (dynamic velocity) with two different speed settings $\left(\left\{v_{o}, v_{c}\right\} \in\{\{5,3\},\{8,4\}\}\right.$ $\mathrm{mm} / \mathrm{s})$.

slight differences in the diagrams were due to differences in the batteries of the robots, although all have a similar capacity (240 mAh). The results demonstrated the success of the proposed perpetual autonomy for a robot swarm system.

\section{Discussion}

\subsection{Temperature Profile}

The recorded temperature during a continuous charging period showed that it increased to $52{ }^{\circ} \mathrm{C}$. This could be reduced by improving ventilation or heat dissipation such as providing an air channel connecting all cells together or heat sinks. Generally, the robots moved continuously on the charging pad and connected to a charging cell for a short span of time depending on their speed. Therefore, each charging cell in the pad's matrix was activated for a few seconds, which did not result in significant heating.

In addition, the robot's battery is placed on top of the robot and it is in room temperature $\left(20^{\circ} \mathrm{C}\right.$ and $\left.25^{\circ} \mathrm{C}\right)$. Hence, the transmitters' heating and the charged during the long-term (12 h) scenarios. The
The last set of experiments in this study was a longterm random walk using three Mona robots. Fig. 17 shows the recorded battery levels for each robot. The 

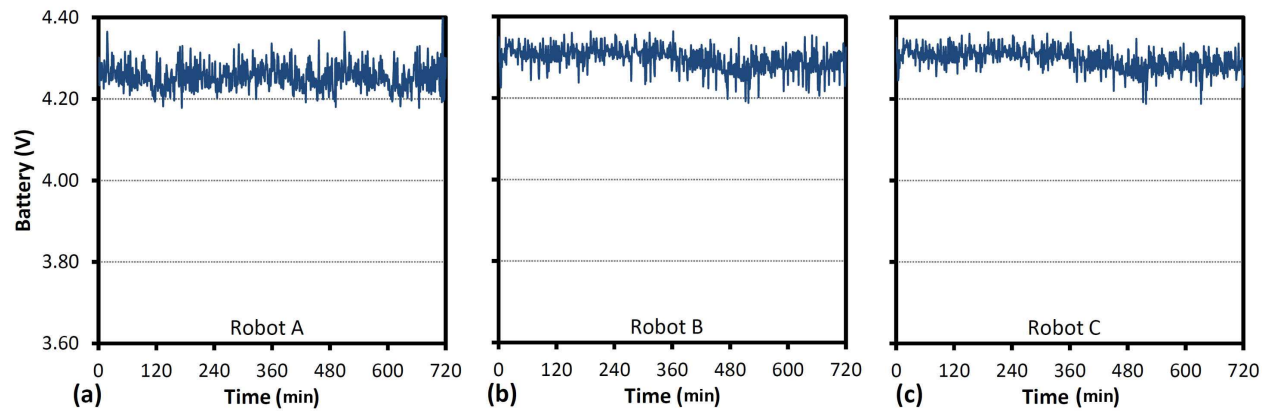

Fig. 17 Recorded battery voltage during 12 hours of multi-robots random walk using an improved walking scenario $\left(v_{o}=8\right.$ $\mathrm{mm} / \mathrm{s}$ and $v_{c}=4 \mathrm{~mm} / \mathrm{s}$ ).

slight heating in the receiver do not impact the robot's operation and its battery.

\subsection{Speed of Motion}

Results of the experiments showed that an increase in speed of the robot increases the swept area during a unit of time which resulted in crossing more charging cells. However, a robot must stay on a transmitter's coil to recharge and increase its battery level. Therefore, increasing the speed of the robot $\left(v_{o}\right)$ reduces the energy harvesting time and also increases power consumption slightly (see Table 2). The trade-off is that the speed of a robot cannot be simply reduced, as it results in prolonging accomplishment time of the main task of a swarm instead of improving the performance.

\subsection{Charging and Discharging Characteristics}

The differences in the discharge characteristics of the individual batteries were revealed as a grey shaded area in Figures 12 to 15. Although all the batteries are Li-Po with the similar capacities, these differences were due to non-homogeneity in the manufacturing of lithium batteries that were reported in $[63,64]$ and they were also related to the low resolution 8-bit ADC module of the deployed microcontroller. However, due to the nature of swarm robotics, these minor heterogeneities in the behaviour of robots are acceptable, since heterogeneity is also observed in the behaviour of social animals. Similar heterogeneities in robot sensory systems and precision of motion were reported in [31]. Note, that the uniform distribution iand proximity of the chargers to each other ensures that the robots crosss the charging pads (and recharge) frequently - typical time between rechargings is 15-30 seconds idepending on the robot speed and situations, where a robot did not recharge for more than a minute were very sparse. Therefore, the robots could potentially use lower capacity batteries than the ones in the experiments performed.

\subsection{Behavioural Improvement}

Performing this set of experiments illustrated that a small adjustment to the walking algorithm could significantly improve the performance of the system. The results showed that the robot's battery level was independent of the duration of the experiment when it utilises a dynamic velocity (varying motion speeds) based on different circumstances. It was a promising result towards a perpetual swarm robotic system. Similar flexibility has been observed in the decision making of insects [65,66] and mammals [67]. Comparable performance improvements have been reported previously. These reports show that several decisioning adjustments based on individual behaviour of a robot swarm resulted in the improved performance of the collective task, such as dynamic velocity and comparative waiting time [54], vector averaging [68], and fuzzy decisioning $[53,69]$.

\subsection{Statistical Analysis}

In most research studies that are involved with physical experiments, clear conclusions cannot be drawn based purely on diagrams and the averages of recorded data. Therefore, there is a need to process all the obtained results with a statistical analysis method. In this paper, the obtained results were analysed with ANOVA to decide the significant factors on the performance of the system. Since all swarm scenarios and battery discharge curves are time-dependent, the time factor $(t)$ is one of the factors in the statistical analysis performed. According to the observed results from the statistical analysis, all the investigated settings (speed of robot, arena configurations, behavioural improvements, and topology of active chargers) have a significant impact on 
the performance of the system. However, the time factor did not have a significant impact in the battery's energy reduction when applying the behavioural improvement.

To find the most significant factor on the performance of system between all the studied configurations, all of the results were analysed together in one test using Tukey Pairwise Comparisons. Table 8 shows the results of grouping information from the Tukey method with $95 \%$ confidence. These groupings mean that those configurations which do not share the same characterisation 'letter' are significantly different.

Table 8 Tukey Pairwise Comparisons of all the studied configurations

\begin{tabular}{l|c|c} 
Configuration & Mean Value & \multicolumn{2}{|l}{ Grouping } \\
\hline Behaviour, 12 Cells & 4.24922 & A \\
Behaviour, 6 Cells & 4.23155 & A \\
\hline Speed, 4 mm/s & 4.07171 & \multicolumn{2}{|c}{ B } \\
12 Active Chargers & 4.07171 & B \\
8 Active Chargers & 4.07119 & \multicolumn{2}{|c}{ B } \\
Topology, CC-a & 4.06076 & B C \\
Topology, CC-b & 4.03625 & \multicolumn{2}{|c}{ C D } \\
Speed, 8 mm/s & 4.02901 & \multicolumn{2}{|c}{ D D } \\
Topology, CC-c & 4.02554 & \multicolumn{2}{|c}{ E } \\
4 Active Chargers & 3.98922 & \multicolumn{2}{|c}{ F }
\end{tabular}

The results revealed a comprehensive conclusion about the most important factors on the performance of the proposed system. It was observed that applying a behavioural improvement (dynamic velocity) clearly made the system independent of the duration of an experiment. It can be seen that the top two factors which impact the system were behavioural improvements (group 'A') even when only $50 \%$ of the chargers were used.

The second level factors (experimental configuration) which significantly impacted the system were shown within a separate group ('B'). It can be seen that the speed of motion and density of chargers were the most important physical factors in this study. By investing in these parameters, which are significantly effective, the performance of the system could be improved. This leads to proposals for several improvements/adjustments on the system including:

- To reduce the delay of the receiver and cell coupling, $t_{c}$, which results in a faster connection between the charger and the robot, to get more benefit in a small span of time, $\delta t$.

- To increase the density of the charger cells allowing robots to cross more chargers after a short gap.

\subsection{Probabilistic Modelling}

The probabilistic model introduced in Section 6 predicted that since the ratio of the effective charging area to the total arena area was lower than the robot consumption/charging power ratio, achieving perpetual operation required that the robot adjusted its behaviour in order to increase the total time spent on the charging pads. In particular, the model correctly predicted that the robot had to reduce its velocity during charging by approximately $40 \%$.

However, the current model does not reflect the inter-robot interactions, which might influence the probability of charging, and therefore, the accuracy of the model's predictions for larger swarms is yet to be verified.

\section{Conclusion and Future Work}

This paper proposed a novel on-the-fly charging system that increased autonomy time of small size mobile robots. This helps research of swarm robotics, allowing implementation of very long duration experiments without frequent interruptions because of battery replacement or recharge. The results showed that behavioural adjustments can improve the performance significantly in comparison to arena configurations, which also had an impact on the performance. A drawback of this system is that recharging the battery in short, discrete time spans may cause a reduction in the long-term battery life. In order to tackle this issue, a recharging management unit is being developed which employs a super-capacitor that is charged at each wireless charging connection. Therefore, the unit will recharge the LiPo battery continuously using the saved energy in the capacitor. The future work is to prepare a large arena $\left(200 \times 80 \mathrm{~cm}^{2}\right)$ with hundreds of charging cells. Based on the predicted values from the proposed probabilistic model, this size of arena can be an ideal platform for large population swarm scenarios.

\section{Acknowledgements}

This work was supported by Innovate UK (Project No. KTP009811), UK EPSRC (Reference: EP/P01366X/1) and Czech Science Foundation project 17-27006Y.

\section{References}

1. K. Gerling, D. Hebesberger, C. Dondrup, T. Körtner, and M. Hanheide, "Robot deployment in long-term care," Zeitschrift für Gerontologie und Geriatrie, vol. 49, no. 4, pp. 288-297, 2016. 
2. D. Hebesberger, T. Koertner, C. Gisinger, J. Pripfl, and C. Dondrup, "Lessons learned from the deployment of a long-term autonomous robot as companion in physical therapy for older adults with dementia: A mixed methods study," in 11th ACM/IEEE International Conference on Human-Robot Interaction (HRI), 2016, pp. 27-34.

3. L. Bayindır, "A review of swarm robotics tasks," Neurocomputing, vol. 172, pp. 292-321, 2016.

4. E. Şahin, "Swarm robotics: From sources of inspiration to domains of application," in International workshop on swarm robotics. Springer, 2004, pp. 10-20.

5. G. Valentini, E. Ferrante, H. Hamann, and M. Dorigo, "Collective decision with 100 kilobots: speed versus accuracy in binary discrimination problems," Autonomous Agents and Multi-Agent Systems, vol. 30, no. 3, pp. 553$580,2016$.

6. F. Arvin, J. C. Murray, L. Shi, C. Zhang, and S. Yue, "Development of an autonomous micro robot for swarm robotics," in IEEE International Conference on Mechatronics and Automation (ICMA), 2014, pp. 635-640.

7. A. E. Turgut, H. Çelikkanat, F. Gökçe, and E. Şahin, "Self-organized Flocking in Mobile Robot Swarms," Swarm Intelligence, vol. 2, no. 2, pp. 97-120, 2008.

8. J. McLurkin, J. Smith, J. Frankel, D. Sotkowitz, D. Blau, and B. Schmidt, "Speaking swarmish: Human-robot interface design for large swarms of autonomous mobile robots," in AAAI spring symposium, 2006.

9. J. M. Santos, T. Krajník, J. P. Fentanes, and T. Duckett, "Lifelong Information-Driven Exploration to Complete and Refine 4-D Spatio-Temporal Maps," IEEE Robotics and Automation Letters, vol. 1, no. 2, pp. 684-691, 2016.

10. M. Bonani, V. Longchamp, S. Magnenat, P. Rétornaz, D. Burnier, G. Roulet, F. Vaussard, H. Bleuler, and F. Mondada, "The marxbot, a miniature mobile robot opening new perspectives for the collective-robotic research," in IROS, 2010.

11. D. Floreano and F. Mondada, "Automatic creation of an autonomous agent: Genetic evolution of a neural network driven robot," in 3rd Int. Conf. on Simulation of Adaptive Behavior: From Animals to Animats 3, 1994.

12. R. A. Watson, S. Ficiei, and J. B. Pollack, "Embodied evolution: Embodying an evolutionary algorithm in a population of robots," in Congress on Evolutionary Computation, 1999.

13. A. F. Winfield and J. Nembrini, "Emergent swarm morphology control of wireless networked mobile robots," in Morphogenetic Engineering, 2012.

14. M. J. Krieger, J.-B. Billeter, and L. Keller, "Ant-like task allocation and recruitment in cooperative robots," Nature, vol. 406, no. 6799 , pp. 992-995, 2000.

15. J. Klingner, A. Kanakia, N. Farrow, D. Reishus, and N. Correll, "A stick-slip omnidirectional drive-train for low-cost swarm robotics: mechanism, calibration, and control," in IROS, 2014.

16. Y. U. Takaya and T. Arita, "Situated and embodied evolution in collective evolutionary robotics," in Int. Symposium on Artificial Life and Robotics, 2003.

17. F. Arvin, T. Krajník, A. E. Turgut, and S. Yue, "COS $\Phi$ : Artificial pheromone system for robotic swarms research," in IROS, Sept 2015.

18. T. Deyle and M. Reynolds, "Surface based wireless power transmission and bidirectional communication for autonomous robot swarms," in ICRA, 2008.

19. M. Karpelson et al., "A wirelessly powered, biologically inspired ambulatory microrobot," in ICRA, 2014.

20. Z. Zhang, X. Xu, B. Li, and B. Deng, "An energyencrypted contactless charging system for swarm robots," in Magnetics Conference (INTERMAG), 2015.
21. G. Caprari, T. Estier, and R. Siegwart, "Fascination of down scaling - alice the sugar cube robot," Journal of Micro-Mechatronics, vol. 1, no. 3, pp. 177-189, 2002.

22. F. Arvin, K. Samsudin, and A. R. Ramli, "Development of a Miniature Robot for Swarm Robotic Application," Int. Jour. of Computer and Electrical Engineering, vol. 1, pp. 436-442, 2009.

23. F. Arvin, J. Murray, C. Zhang, and S. Yue, "Colias: An Autonomous Micro Robot for Swarm Robotic Applications," International Journal of Advanced Robotic Systems, vol. 11, no. 113, pp. 1-10, 2014.

24. F. Mondada, M. Bonani, X. Raemy, J. Pugh, C. Cianci, A. Klaptocz, S. Magnenat, J.-C. Zufferey, D. Floreano, and A. Martinoli, "The e-puck, a robot designed for education in engineering," in 9th conference on autonomous robot systems and competitions, 2009.

25. M. Dorigo, D. Floreano, L. M. Gambardella, F. Mondada, S. Nolfi, T. Baaboura, M. Birattari, M. Bonani, M. Brambilla, A. Brutschy et al., "Swarmanoid: a novel concept for the study of heterogeneous robotic swarms," IEEE Robotics \& Automation Magazine, vol. 20, no. 4, pp. 60-71, 2013.

26. S. Kernbach, R. Thenius, O. Kernbach, and T. Schmickl, "Re-embodiment of Honeybee Aggregation Behavior in an Artificial Micro-Robotic System," Adaptive Behavior, vol. 17, no. 3, pp. 237-259, 2009.

27. F. Mondada, E. Franzi, and P. Ienne, "Mobile robot miniaturisation: A tool for investigation in control algorithms," in Experimental robotics III, 1994.

28. M. Rubenstein, C. Ahler, N. Hoff, A. Cabrera, and R. Nagpal, "Kilobot: A low cost robot with scalable operations designed for collective behaviors," Robotics and Autonomous Systems, vol. 62, no. 7, pp. 966-975, 2014.

29. F. Mondada, G. C. Pettinaro, A. Guignard, I. W. Kwee, D. Floreano, J.-L. Deneubourg, S. Nolfi, L. M. Gambardella, and M. Dorigo, "Swarm-bot: A new distributed robotic concept," Autonomous robots, vol. 17, no. 2-3, pp. 193-221, 2004

30. G. Caprari and R. Siegwart, "Mobile micro-robots ready to use: Alice," in IEEE/RSJ International Conference on Intelligent Robots and Systems. IEEE, 2005, pp. 32953300.

31. T. Schmickl, R. Thenius, C. Moeslinger, G. Radspieler, S. Kernbach, M. Szymanski, and K. Crailsheim, "Get in touch: cooperative decision making based on robotto-robot collisions," Autonomous Agents and Multi-Agent Systems, vol. 18, no. 1, pp. 133-155, 2009.

32. W. G. Walter, The living brain. New York: Norton, 1953.

33. S. Yuta and Y. Hada, "Long term activity of the autonomous robot-proposal of a bench-mark problem for the autonomy," in IEEE/RSJ International Conference on Intelligent Robots and Systems, vol. 3, 1998, pp. 1871-1878.

34. K. Roufas, Y. Zhang, D. Duff, and M. Yim, "Six degree of freedom sensing for docking using ir led emitters and receivers," in Experimental Robotics VII. Springer, 2001, pp. 91-100.

35. B. W. Minten, R. R. Murphy, J. Hyams, and M. Micire, "Low-order-complexity vision-based docking," IEEE Transactions on Robotics and Automation, vol. 17, no. 6, pp. 922-930, 2001.

36. T. Krajník, M. Nitsche, J. Faigl, P. Vaněk, M. Saska, L. Přeučil, T. Duckett, and M. Mejail, "A Practical Multirobot Localization System," Journal of Intelligent $\&$ Robotic Systems, vol. 76, no. 3-4, pp. 539-562, 2014. 
37. F. Arvin, K. Samsudin, and A. R. Ramli, "Swarm robots long term autonomy using moveable charger," in International Conference on Future Computer and Communication. IEEE, 2009, pp. 127-130.

38. A. Attarzadeh, "Development of advanced power management for autonomous micro-robots," Master's thesis, University of Stuttgart, Germany, 2006.

39. A. Martinoli, E. Franzi, and O. Matthey, "Towards a reliable set-up for bio-inspired collective experiments with real robots," in Experimental Robotics V. Springer, 1998, pp. 595-608.

40. X. Wei, Z. Wang, and H. Dai, "A critical review of wireless power transfer via strongly coupled magnetic resonances," Energies, vol. 7, no. 7, pp. 4316-4341, 2014.

41. X. Lu, P. Wang, D. Niyato, D. I. Kim, and Z. Han, "Wireless charging technologies: Fundamentals, standards, and network applications," IEEE Communication Surveys 8 Tutorials, vol. 18, pp. $1413-1452,2016$.

42. — - "Wireless networks with RF energy harvesting: A contemporary survey," IEEE Communication Surveys \& Tutorials, vol. 17, no. 2, pp. $757-789,2015$.

43. B. Cannon, J. Hoburg, D. Stancil, and S. Goldstein., "Magnetic resonant coupling as a potential means for wireless power transfer to multiple small receivers," IEEE Trans. Power Electron., vol. 24, no. 7, pp. 1819 - 1825, 2009.

44. C. J. Chen, T. H.Chu, C. L. Lin, and Z. C. Jou, "A study of loosely coupled coils for wireless power transfer," IEEE Trans. Circuitsand Syst.Part II: Express Briefs, vol. 57, no. 7 , pp. $536-540,2010$.

45. W. Zhong, C. K. Lee, and S. Y. Hui, "Wireless power domino-resonator systems with noncoaxial axes and circular structures," IEEE Transactions on Power Electronics, vol. 27, no. 11, pp. 4750-4762, 2012.

46. C. Hu, F. Arvin, C. Xiong, and S. Yue, "A Bio-inspired Embedded Vision System for Autonomous Micro-robots: the LGMD Case," IEEE Transactions on Cognitive and Developmental Systems, vol. 9, no. 3, pp. 241-254, 2017.

47. F. Arvin and M. Bekravi, "Encoderless Position Estimation and Error Correction Techniques for Miniature Mobile Robots," Turkish Journal of Electrical Engineering \& Computer Sciences, vol. 21, pp. 1631-1645, 2013.

48. F. Arvin, K. Samsudin, and A. R. Ramli, "Development of IR-Based Short-Range Communication Techniques for Swarm Robot Applications," Advances in Electrical and Computer Engineering, vol. 10, no. 4, pp. 61-68, 2010.

49. T. Krajník, M. Nitsche, J. Faigl, T. Duckett, M. Mejail, and L. Přeučil, "External localization system for mobile robotics," in 16th International Conference on Advanced Robotics (ICAR). IEEE, 2013, pp. 1-6.

50. F. A. Ahmad, A. R. Ramli, K. Samsudin, and S. J. Hashim, "Optimization of power utilization in multimobile robot foraging behavior inspired by honeybees system," The Scientific World Journal, vol. 2014, pp. 1-12, 2014.

51. F. Arvin, A. E. Turgut, T. Krajník, and S. Yue, "Investigation of cue-based aggregation in static and dynamic environments with a mobile robot swarm," Adaptive Behavior, vol. 24, no. 2, pp. 102-118, 2016.

52. E. Ferrante, A. E. Turgut, E. Duéñez-Guzmán, M. Dorigo, and T. Wenseleers, "Evolution of selforganized task specialization in robot swarms," PLoS Comput Biol, vol. 11, no. 8, p. e1004273, 2015.

53. F. Arvin, A. E. Turgut, F. Bazyari, K. B. Arikan, N. Bellotto, and S. Yue, "Cue-based aggregation with a mobile robot swarm: a novel fuzzy-based method," Adaptive Behavior, vol. 22, no. 3, pp. 189-206, 2014.
54. F. Arvin, K. Samsudin, A. R. Ramli, and M. Bekravi, "Imitation of Honeybee Aggregation with Collective Behavior of Swarm Robots," International Journal of Computational Intelligence Systems, vol. 4, no. 4, pp. 739$748,2011$.

55. J. Neter, M. H. Kutner, C. J. Nachtsheim, and W. Wasserman, Applied linear statistical models. Irwin Chicago, 1996, vol. 4.

56. N. Correll and A. Martinoli, "Modeling self-organized aggregation in a swarm of miniature robots," in IEEE International Conference on Robotics and Automation, Workshop on Collective Behaviors Inspired by Biological and Biochemical Systems, 2007.

57. A. Martinoli, A. Ijspeert, and F. Mondada, "Understanding collective aggregation mechanisms: From probabilistic modelling to experiments with real robots," Robotics and Autonomous Systems, vol. 29, no. 1, pp. 51-63, 1999.

58. O. Soysal and E. Şahin, "A macroscopic model for selforganized aggregation in swarm robotic systems," in Swarm Robotics, ser. Lecture Notes in Computer Science, E. Şahin, W. Spears, and A. Winfield, Eds., 2007, vol. 4433, pp. 27-42.

59. L. Bayindir and E. Şahin, "Modeling self-organized aggregation in swarm robotic systems," in Swarm Intelligence Symposium. IEEE, 2009, pp. 88-95.

60. H. Hamann, "Space-time continuous models of swarm robotics systems: Supporting global-to-local programming," Ph.D. dissertation, Department of Computer Science, University of Karlsruhe, 2008.

61. T. Schmickl, H. Hamann, H. Worn, and K. Crailsheim, "Two different approaches to a macroscopic model of a bio-inspired robotic swarm," Robotics and Autonomous Systems, vol. 57, no. 9, pp. 913-921, 2009.

62. F. Arvin, A. Attar, A. Turgut, and S. Yue, "Powerlaw distribution of long-term experimental data in swarm robotics," in Advances in Swarm and Computational Intelligence, ser. Lecture Notes in Computer Science, Y. Tan, Y. Shi, F. Buarque, A. Gelbukh, S. Das, and A. Engelbrecht, Eds., 2015, vol. 9140, pp. 551-559.

63. P. Taheri, A. Mansouri, B. Schweitzer, M. Yazdanpour, and M. Bahrami, "Electrical constriction resistance in current collectors of large-scale lithium-ion batteries," Journal of The Electrochemical Society, vol. 160, no. 10, pp. A1731-A1740, 2013.

64. A. Senyshyn, M. Mühlbauer, O. Dolotko, M. Hofmann, and H. Ehrenberg, "Homogeneity of lithium distribution in cylinder-type li-ion batteries," Scientific reports, vol. 5, p. $18380,2015$.

65. S. Garnier, J. Gautrais, M. Asadpour, C. Jost, and G. Theraulaz, "Self-Organized Aggregation Triggers Collective Decision Making in a Group of Cockroach-Like Robots," Adaptive Behavior, vol. 17, no. 2, pp. 109-133, 2009.

66. R. Jeanson, C. Rivault, J.-L. Deneubourg, S. Blanco, R. Fournier, C. Jost, and G. Theraulaz, "Self-organized Aggregation in Cockroaches," Animal Behaviour, vol. 69, no. 1 , pp. $169-180,2005$.

67. H. Liwanag, J. Oraze, D. Costa, and T. Williams, "Thermal benefits of aggregation in a large marine endotherm: huddling in california sea lions," Journal of Zoology, vol. 293, no. 3, pp. 152-159, 2014.

68. F. Arvin, A. E. Turgut, N. Bellotto, and S. Yue, "Comparison of different cue-based swarm aggregation strategies," in Advances in Swarm Intelligence, ser. Lecture Notes in Computer Science, vol. 8794, 2014, pp. 1-8.

69. F. Arvin, A. E. Turgut, and S. Yue, "Fuzzy-Based Aggregation with a Mobile Robot Swarm," in Swarm 
Intelligence, ser. Lecture Notes in Computer Science, vol. 7461, 2012, pp. 346-347.

\section{Appendix I}

In this work, inductive charging circuits were developed using wireless power transfer module BQ500210 and wireless power receiver module BQ51013B. The receiver and transmitter circuits were designed as the typical application circuit proposed in the modules' datasheets. Figure 18 shows schematics of the transmitter and receiver, which were used in the work presented. 


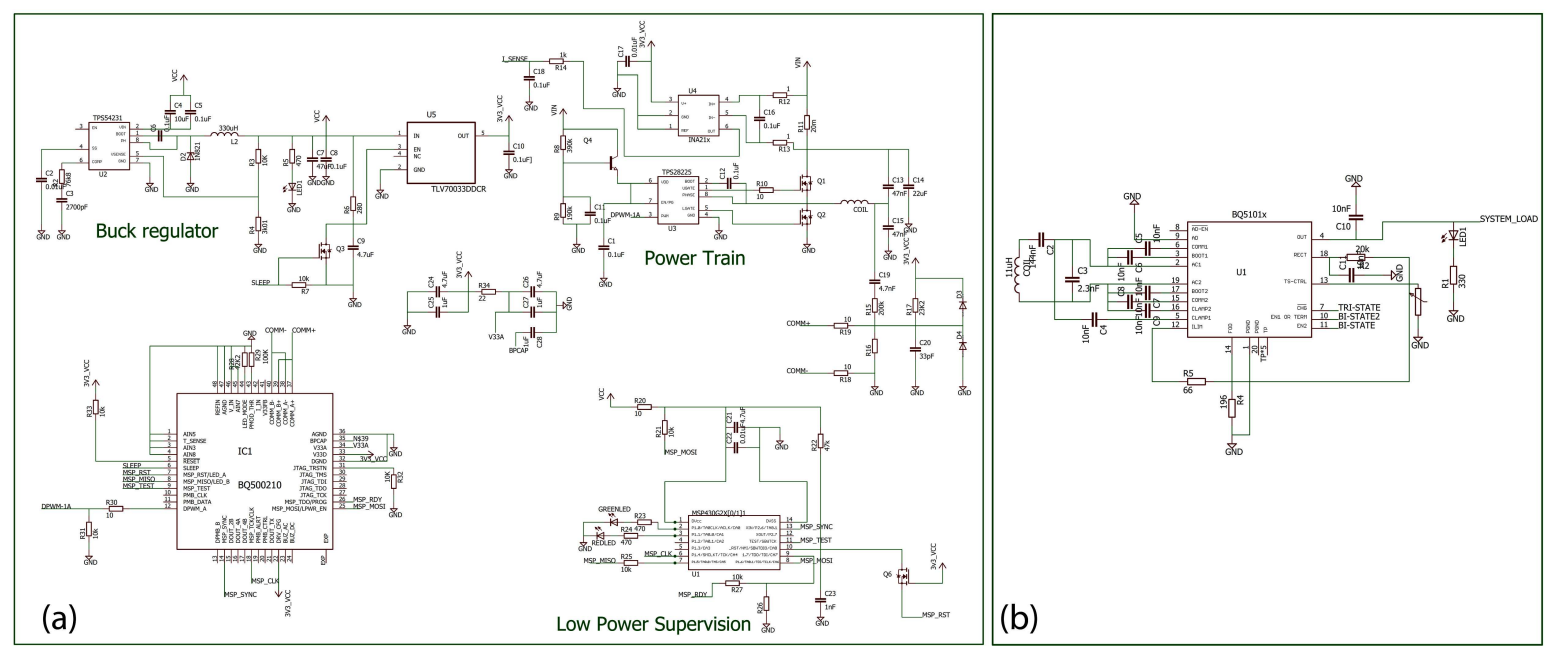

Fig. 18 Schematics of (a) transmitter and (b) receiver used in this work. Schematics is based on the simplified diagram in datasheets of the modules' manufacturer (www.ti.com). 